\title{
Collective modes in superfluid helium when there is a relative velocity between the normal and superfluid components
}

\author{
I.N. Adamenko, K.E. Nemchenko, and V.A. Slipko \\ V.N. Karazin Kharkov National University, 61077, Ukraine \\ E-mail: vaslipko@mail.ru \\ A.F.G. Wyatt \\ School of Physics, University of Exeter, Exeter, EX4 4QL, United Kingdom \\ E-mail: A.F.G.Wyatt@exeter.ac.uk
}

Received November 6, 2007

\begin{abstract}
Collective modes are studied in superfluid helium when the normal and superfluid components have a relative velocity $\mathbf{w}$. In this paper the general dispersion relation for first and second sound modes is obtained for arbitrary values of $\mathbf{w}$, and we have found the relationship between the amplitudes of the oscillating variables for first sound. It is shown in a first sound wave, that both temperature and pressure can oscillate, and moreover, the normal fluid velocity can exceed the superfluid velocity in the wave. In the general case of first sound, the normal fluid not only has a velocity component parallel to the wave vector, but also a transverse velocity component. It is shown that when there is only a phonon system in the helium, the amplitude of the temperature oscillation in a first sound wave in an anisotropic phonon system, can exceed that in a second sound wave in an isotropic phonon system, for similar values of the normal fluid density.
\end{abstract}

PACS: 67.25.D- Superfluid phase;

67.25.dt Sound and excitations.

Keywords: normal and superfluid components, first and second sound, phonon system in helium.

\section{Introduction}

In superfluid helium systems with high values of the relative velocity of the normal and superfluid components are studied experimentally in various ways. One of them uses pulses of thermal excitations in helium [1]. Such pulses are created by a heater immersed into helium at very low temperature when a short electrical current is applied to the heater. The heater then radiates excitations into the helium. Another way to create a large relative velocity is to make the helium move through narrow channels. In these systems, the velocities of the normal or superfluid components can be very close to the critical velocity. Such systems have attracted the attention of both theoreticians [2-4] and experimentalists [5-9].

The two-fluid hydrodynamic equations of superfluid helium cause a fundamental difference between helium and classical liquids. The processes that can take place in pulses of classical gases, or in moving liquids, has in principle the same properties as processes in a stationary gas or liquid. This is due to the fact that the coordinate frame can be transformed into a frame where the gas pulse, or moving liquid, is stationary.

In superfluid helium the situation is very different. If the relative velocity $\mathbf{w}=\mathbf{v}_{n}-\mathbf{v}_{s}$, where $\mathbf{v}_{n}$ and $\mathbf{v}_{s}$ are the normal and superfluid velocities, is not equal to zero, then it is impossible to find a frame where velocities of both components of helium are equal to zero. This fact means that most phenomena will have different properties in superfluid helium and, moreover, new properties compared to stationary helium when $\mathbf{w}=0$.

The theoretical description of these problems was first made by [10]. There second sound propagation was analyzed at very small values of velocity $\mathbf{w}$. Results on second sound, at arbitrary values of $\mathbf{w}$, were presented in [11] where the dispersion equation for second sound was obtained with the approximations that the coefficient of thermal expansion, and momentum density of helium, are equal to zero. These approximations are not valid in the general case. 
In Refs. 2 and 12, as well as in Ref. 3 the temperature dependence of the critical velocity of motion in helium $\mathbf{w}_{c}(T)$ was studied. This allowed us to determine the limits of thermodynamic stability in helium. In Refs. 3 and 4 it was shown that quasiparticle system, with large values of $\mathbf{w}$, possesses unique thermodynamic properties.

In this paper, the general dispersion relation for first and second sounds, at arbitrary values of $\mathbf{w}$, is obtained. We have obtained the relationship between the amplitudes of the hydrodynamic variables for first sound. The results obtained in this paper are significantly different from the well-known results which are obtained for isotropic phonon system $(\mathbf{w}=0)$ in superfluid helium.

\section{Sound modes in superfluid helium}

The hydrodynamic equations for superfluid helium according to Refs. 13, 14 can be written as follows:

$$
\begin{gathered}
\frac{\partial \rho}{\partial t}+\operatorname{div}\left(\rho_{n} \mathbf{v}_{n}+\rho_{s} \mathbf{v}_{s}\right)=0 \\
\frac{\partial S}{\partial t}+\operatorname{div}\left(S \mathbf{v}_{n}\right)=0 \\
\frac{\partial \mathbf{v}_{S}}{\partial t}+\nabla \mu+\left(\mathbf{v}_{S} \nabla\right) \mathbf{v}_{s}=0 \\
\frac{\partial A_{i}}{\partial t}+\left(\mathbf{v}_{n} \nabla\right) A_{i}=-\frac{\partial T}{\partial x_{i}}-A_{k} \frac{\partial v_{n k}}{\partial x_{i}} .
\end{gathered}
$$

Here $\rho$ is the density of helium; $\rho_{n}$ and $\rho_{s}$ are densities of the normal and superfluid components, respectively; $\mathbf{v}_{n}$ and $\mathbf{v}_{S}$ are velocities of normal and superfluid components, respectively; $S$ is entropy of unit of volume; $\mu$ is the chemical potential of unit of mass of helium, $\mathbf{A}=\rho_{n} \mathbf{w} / S$; $\mathbf{w}=\mathbf{v}_{n}-\mathbf{v}_{S}$ is the relative velocity of the normal and superfluid components. After linearization of this system of equations, we consider the solution, for small deviations, as plane waves in an infinite medium:

$$
\begin{gathered}
\left(v_{s \|}-u\right) \widetilde{\rho}+w_{\|} \widetilde{\rho}_{n}+\rho_{n} \widetilde{v}_{n \|}+\rho_{s} \widetilde{v}_{s \|}=0 \\
\left(v_{n \|}-u\right) \widetilde{S}+\widetilde{v}_{n \|}=0 \\
\left(v_{s \|}-u\right) \widetilde{\mathbf{v}}_{s}+\widetilde{\mu} \frac{\mathbf{k}}{k}=0, \\
\left(v_{n \|}-u\right)\left[\tilde{\mathbf{w}}+\mathbf{w}\left(\frac{\widetilde{\rho}_{n}}{\rho_{n}}-\frac{\tilde{S}}{S}\right)\right]+\left(\frac{S}{\rho_{n}} \widetilde{T}+\mathbf{w} \widetilde{\mathbf{v}}_{n}\right) \frac{\mathbf{k}}{k}=0 .
\end{gathered}
$$

Here $v_{s \|}=\mathbf{v}_{s} \mathbf{k} / k$, and similarly for $v_{n \|}$ and $w_{\|}$. Vector $\mathbf{k}$ is the wave vector and $k$ its modulus, $u$ is the modulus of the phase velocity and $u=\omega / k$, where $\omega$ is the angular frequency of the mode. Small deviations of the parameters are marked with the symbol «tilde». From Eq. (7) it follows that the oscillation of the superfluid velocity is always longitudinal if $u \neq v_{s \|}$. The reason that the collective mode with $u=v_{s \|}$ does not exist, is because the superfluid

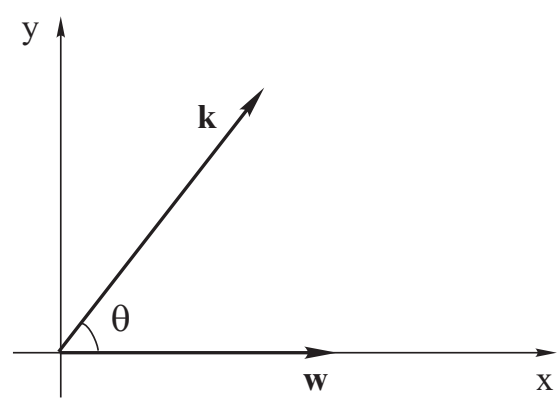

Fig. 1. The coordinate frame with the $x$ axis directed along $\mathbf{w}$, and with the $y$ axis lying in the plane which defined by vector $\mathbf{w}$ and wave vector $\mathbf{k}$. The angle $\theta$ is between vectors $\mathbf{k}$ and $\mathbf{w}$.

always has potential flow. At the same time from Eq. (8) it follows that the oscillation of the relative velocity $\widetilde{\mathbf{w}}$ and the normal fluid velocity $\widetilde{\mathbf{v}}_{n}$, have in general both longitudinal and transverse components relative to the wave vector $\mathbf{k}$.

We choose the coordinate frame, with axis $x$ directed along the vector of the relative velocity $\mathbf{w}$, axis $y$ lies in the plane determined by the vectors $\mathbf{w}$ and $\mathbf{k}$ and $k_{y}>0$ (see Fig. 1). Equations (5)-(8) are a system of five equations for any two scalar variables, for example, pressure $\widetilde{P}$ and temperature $\widetilde{T}$, and the superfluid velocity $\widetilde{v}_{S \|}$ (its projection on vector $\mathbf{k}$ ) and two components of the vector of relative velocity $\widetilde{\mathbf{w}}$.

Now we should take into account the thermodynamic equations:

$$
\begin{aligned}
& \widetilde{\rho}=\left(\frac{\partial \rho}{\partial P}\right)_{T, w} \widetilde{P}+\left(\frac{\partial \rho}{\partial T}\right)_{P, w} \widetilde{T}+\left(\frac{\partial \rho}{\partial\left(w^{2} / 2\right)}\right)_{P, T} \mathbf{w} \widetilde{\mathbf{w}} ; \quad(9) \\
& \widetilde{\rho}_{n}=\left(\frac{\partial \rho_{n}}{\partial P}\right)_{T, w} \widetilde{P}+\left(\frac{\partial \rho_{n}}{\partial T}\right)_{P, w} \widetilde{T}+\left(\frac{\partial \rho_{n}}{\partial\left(w^{2} / 2\right)}\right)_{P, T} \mathbf{w} \widetilde{\mathbf{w}}
\end{aligned}
$$

$$
\widetilde{S}=\left(\frac{\partial S}{\partial P}\right)_{T, w} \widetilde{P}+\left(\frac{\partial S}{\partial T}\right)_{P, w} \widetilde{T}+\left(\frac{\partial S}{\partial\left(w^{2} / 2\right)}\right)_{P, T} \mathbf{w} \tilde{\mathbf{w}}
$$

and the relation for the chemical potential

$$
d \mu=\frac{1}{\rho} d P-\frac{S}{\rho} d T-\frac{\rho_{n}}{\rho} \mathbf{w} d \mathbf{w},
$$

which gives a connection between the small deviations

$$
\widetilde{\mu}=\frac{1}{\rho} \widetilde{P}-\frac{S}{\rho} \widetilde{T}-\frac{\rho_{n}}{\rho} \mathbf{w} \widetilde{\mathbf{w}},
$$

and determines the relations between the derivatives of the thermodynamic variables:

$$
\frac{\partial}{\partial T}\left(\frac{1}{\rho}\right)_{P, w}=-\frac{\partial}{\partial P}\left(\frac{S}{\rho}\right)_{T, w}
$$




$$
\begin{aligned}
& \frac{\partial}{\partial\left(w^{2} / 2\right)}\left(\frac{S}{\rho}\right)_{P, T}=\frac{\partial}{\partial T}\left(\frac{\rho_{n}}{\rho}\right)_{P, w} ; \\
& \frac{\partial}{\partial\left(w^{2} / 2\right)}\left(\frac{1}{\rho}\right)_{P, T}=-\frac{\partial}{\partial P}\left(\frac{\rho_{n}}{\rho}\right)_{T, w} .
\end{aligned}
$$

The system (5)-(8) has a nontrivial solution, for any value of $\mathbf{w}$, if its determinant is equal to zero. This condition gives an equation of fifth order with respect to the phase velocity $u$.

The dispersion equation for one of the modes can be easily found directly from the system of Eqs. (5)-(8). This is because the vector Eq. (8) becomes a scalar equation when $u=v_{n \|}$. So, a nontrivial solution of Eqs. (5)-(8) should exist if $u=v_{n \|}=\mathbf{v}_{n} \mathbf{k} / k$. We call this mode the transverse mode and have analyzed it in Ref. 15.

\section{Dispersion equation for first and second sounds at arbitrary values of relative velocity}

To obtain the dispersion relation for first and second sound, we can start from the Eqs. (5)-(8) with $u \neq v_{n \|}$, which explicitly excludes the transverse mode. So we take the expression for $\widetilde{v}_{n \|}$ from (6) and for $\widetilde{v}_{s \|}$ from (7) and put them into Eq. (5). Finally we get the equation

$\tilde{\mu}=\left(u-v_{s \|}\right)\left[\left(u-v_{s \|}\right) \frac{\tilde{\rho}}{\rho_{s}}-w_{\|} \frac{\tilde{\rho}_{n}}{\rho_{s}}-\left(u-v_{n \|}\right) \frac{\rho_{n}}{\rho_{s}} \frac{\tilde{S}}{S}\right]$.

Then we get for the $y$ component of relative velocity from Eq. (8)

$$
\widetilde{w}_{y}=\frac{\sin \theta}{u-v_{n \|}}\left[\frac{S}{\rho_{n}} \widetilde{T}+w \widetilde{w}_{x}+\frac{w_{\|}}{u-v_{s \|}} \widetilde{\mu}\right] .
$$

For the $x$ component of Eq. (8) and the amplitude of the temperature oscillation, we obtain following equations:

$$
\begin{gathered}
\widetilde{w}_{x}+\cos \theta\left(u-v_{s \|}\right) \frac{\tilde{\rho}}{\rho_{s}}+w \sin ^{2} \theta\left(\frac{\widetilde{\rho}_{n}}{\rho_{n}}-\frac{\widetilde{S}}{S}\right)- \\
-w \cos ^{2} \theta \frac{\widetilde{\rho}_{n}}{\rho_{s}}-\cos \theta\left(u-v_{n \|}\right) \frac{\rho}{\rho_{s}} \frac{\widetilde{S}}{S}=0, \\
\cos \theta \frac{S}{\rho_{n}} \widetilde{T}-\left(u-v_{n \|}\right) \widetilde{w}_{x}+w \cos ^{2} \theta\left(u-v_{n \|}\right) \frac{\widetilde{S}}{S}+ \\
+w\left[u-v_{n \|}+w \cos \theta \sin ^{2} \theta\right]\left(\frac{\widetilde{S}}{S}-\frac{\widetilde{\rho}_{n}}{\rho_{n}}\right)=0 .
\end{gathered}
$$

Using relation (13), for small deviations of the chemical potential, in the Eq. (17) we find

$$
\begin{aligned}
& \frac{1}{\rho} \widetilde{P}-\frac{S}{\rho} \widetilde{T}-\frac{\rho_{n}}{\rho} w \widetilde{w}_{x}+w \cos \theta\left(u-v_{s \|}\right) \frac{\widetilde{\rho}_{n}}{\rho_{s}}+ \\
& +\left(u-v_{s \|}\right)\left(u-v_{n \|}\right) \frac{\rho_{n}}{\rho_{s}} \frac{\widetilde{S}}{S}-\left(u-v_{s \|}\right)^{2} \frac{\widetilde{\rho}}{\rho_{s}}=0 .
\end{aligned}
$$

The system of Eqs. (19)-(21) together with thermodynamic relations (9)-(11) form a system of three equations for the three independent variables $\widetilde{P}, \widetilde{T}, \widetilde{w}_{x}$. For a nontrivial solution of this system of equations to exist, the determinant from these equations must be zero. This gives a fourth order equation for $u$, since Eqs. (19) and (20) are linear and Eq. (21) is quadratic with respect to $u$. As a result, we get the dispersion relation as an algebraic expression of the fourth power. This is explicitly presented in Appendix A. The four roots of this dispersion relation are the two modes of first sound and the two modes of second sound, at arbitrary values of the relative velocity $w$.

In the limiting case of $w=0$, the general relations (A.1)-(A.6) give the results for the velocities of sound obtained in Ref. 13. In the case of small values of $w$, in the linear approximation and neglecting thermal expansion $\alpha$, the general relations (A.1)-(A.6) give the result in Ref. 10, obtained there for the case $w<<c$ and $\alpha=0$.

\section{First sound at arbitrary values of relative velocity}

Consider the first sound mode in the important practical case when $\rho_{n}<<\rho$, and $\rho_{s} \approx \rho$ at arbitrary values of $w$. This case, in particular, occurs in experiments $[1,5,8,9]$.

We choose the coordinate frame where $\mathbf{j}=0$. This can be always obtained by the appropriate Galilean transformation. Using the inequality $\rho_{n}<<\rho$, in this frame we get

$$
\mathbf{v}_{n} \approx \mathbf{w}, \quad \mathbf{v}_{s} \approx 0 \text {. }
$$

At relatively low temperatures, when $\rho_{n}<<\rho$, the general dispersion relation (see Appendix A) has a solution

$$
u= \pm c
$$

for first sound.

To get the relation between the oscillating variables, we start from the initial system of Eqs. (19)-(21), where we substitute the dispersion relation (23) and take into account Eq. (22) and condition $\rho_{n}<<\rho$. As the result, we get the following expressions for the mode $u=+c$ :

$$
\begin{gathered}
\widetilde{w}_{x}=\frac{C}{D} \frac{S}{\rho_{n}} \frac{\widetilde{P}}{\rho c}, \\
\widetilde{T}=\frac{E}{D} \frac{\widetilde{P}}{\rho c} .
\end{gathered}
$$

Here we introduce variables $C, D$, and $E$ defined in Appendix B. 
For the mode $u=-c$, the relations can be obtained by substituting, in Eqs. (24) and (25), the angle $\pi-\theta$ for $\theta$, which corresponds to substituting $-u$ for $u$. By projecting the oscillation of superfluid velocity onto the vector $\mathbf{k}$, and with Eq. (7) and relations (22), we get for the first sound modes $u= \pm c$ :

$$
\widetilde{v}_{s}=\frac{\tilde{\mu}}{u-v_{s \|}} \approx \frac{1}{u} \tilde{\mu} \approx \frac{\widetilde{P}}{\rho u},
$$

where we have used, in accordance with Eq. (13) or (22), the approximate equality

$$
\widetilde{\mu} \approx \frac{\widetilde{P}}{\rho},
$$

which applies for first sound because the contribution of thermal excitations to $\tilde{\mu}$ can be neglected as $\rho_{n}<<\rho$.

Substituting expressions (24), (25), and (27) into Eq. (18) gives the projection of the oscillation of the relative velocity onto the axis that is perpendicular to vector $\mathbf{w}, \widetilde{w}_{y}$, for the first sound mode with $u=+c$ :

$$
\widetilde{w}_{y}=\frac{\sin \theta\left[w \cos \theta D+\frac{S}{\rho_{n}}(E+w C)\right]}{(c-w \cos \theta) D} \frac{\widetilde{P}}{\rho c} .
$$

For the mode $u=-c$, we substitute $\pi-\theta$ for $\theta$ in Eq. (28) and multiply the right hand side by -1 .

The relations (24)-(28) determine the oscillation of the main thermodynamic variables, the superfluid and the relative velocity components, for the first sound modes $u= \pm c$. This case is very important in practice as it is the case where the contribution of the thermal excitations is negligible $\left(\rho_{n}<<\rho\right)$ and the relative velocity can take any value.

In the limit $w=0$, from Eqs. (B.1)-(B.3) we get

$$
\begin{gathered}
C=\frac{\cos \theta}{c} E=\frac{S}{\rho_{n}}\left[\rho c^{2}\left(\frac{\partial \ln S}{\partial P}\right)_{T, w}-1\right] \cos \theta= \\
=\frac{c^{2}}{\rho_{n}}\left(\frac{\partial \rho}{\partial T}\right)_{P, w} \cos \theta, \\
D=\frac{S}{\rho_{n}}\left[\frac{S}{\rho_{n}}-\frac{\partial \ln S}{\partial T} c^{2}\right],
\end{gathered}
$$

where we use the equality (14) for the transformation of the thermodynamic derivatives. In the limit $w=0$, the oscillations of all vector values are longitudinal (i.e., along k), so, we can take $\theta=0$ in (29). Substituting (29) and (30) into (24) and (25) we get, using (26), the relations between the amplitudes of the oscillating variables. These coincide with the result of Ref. 13. At $w=0$ there is no difference between modes $u= \pm c$. For first sound, at $w=0$, pressure and the normal and superfluid velocities oscillate, and the temperature oscillations are determined by the small value of the coefficient of thermal expansion in helium. As will be shown below, an unusual situation appears when $w$ is not small.

\section{Collective mode of first sound in a phonon system at arbitrary values of $w$}

Let us consider a phonon system when we only have phonons in the superfluid helium. For example, helium at $T<0.6 \mathrm{~K}$ for the isotropic case, and a phonon pulse in helium at $T \leq 0.05 \mathrm{~K}$ in the anisotropic case.

The general relations can be written explicitly for the phonon system with linear dispersion:

$$
\varepsilon=c p
$$

where $c^{2}=\partial P / \partial \rho$ is the sound velocity of liquid helium. In this case [13]

$$
\frac{S}{\rho_{n}}=\frac{c^{2}-w^{2}}{T},
$$

and

$$
\rho_{n}=\frac{2 \pi^{2}}{45} \frac{T^{4}}{\hbar^{3} c^{5}\left(1-w^{2} / c^{2}\right)^{3}} .
$$

Calculating the respective derivatives for the phonon system, we get

$$
\begin{gathered}
\left(\frac{\partial \ln \rho_{n}}{\partial P}\right)_{T, w}=\frac{\partial}{\partial P}\left[\ln \frac{c}{\left(c^{2}-w^{2}\right)^{3}}\right]_{T, w}=-\frac{5 c^{2}+w^{2}}{c^{2}-w^{2}} \frac{u_{G}}{\rho c^{2}}, \\
\left(\frac{\partial \ln S}{\partial P}\right)_{T, w}=-\frac{3 c^{2}+w^{2}}{c^{2}-w^{2}} \frac{u_{G}}{\rho c^{2}},
\end{gathered}
$$

where

$$
u_{G}=\frac{\rho}{c} \frac{\partial c}{\partial \rho}=2.84
$$

is the Gruneisen constant. Using these expressions for the thermodynamic derivatives we get from Eqs. (19)-(21) the following expressions for the amplitudes of the first sound mode $u=+c$ in a phonon system:

$$
\begin{aligned}
& \widetilde{w}_{x}=\left\{2 w^{2}\left(w^{2}-c^{2}\right) \cos ^{3} \theta+c w\left[3 w^{2}\left(u_{G}-1\right)+c^{2}\left(3-u_{G}\right)\right] \cos ^{2} \theta+\right. \\
& \left.+\left[w^{4}\left(u_{G}+1\right)-2 w^{2} c^{2}\left(4 u_{G}+1\right)+c^{4}\left(3 u_{G}+1\right)\right] \cos \theta+c w\left[\left(u_{G}+1\right) w^{2}+c^{2}\left(u_{G}-1\right)\right]\right\} \frac{1}{2 c^{2}(c-w \cos \theta)^{2}} \frac{\widetilde{P}}{\rho c}
\end{aligned}
$$




$$
\begin{aligned}
& \frac{\widetilde{T}}{T}=\left\{2 w^{3} \cos ^{3} \theta+2 c w^{2}\left(u_{G}-2\right) \cos ^{2} \theta+w\left[u_{G} w^{2}+w^{2}-\left(7 u_{G}+1\right) c^{2}\right] \cos \theta+c\left(c^{2}+w^{2}\right)+c u_{G}\left(w^{2}+3 c^{2}\right)\right\} \times \\
& \times \frac{1}{2 c^{2}(c-w \cos \theta)^{2}} \frac{\widetilde{P}}{\rho c} \cdot \\
& \widetilde{w}_{y}=\left\{-2 w^{2} c \cos ^{2} \theta+w\left[\left(3-u_{G}\right) c^{2}-\left(1+u_{G}\right) w^{2}\right] \cos \theta+c^{3}\left(3 u_{G}+1\right)-c w^{2}\left(u_{G}+1\right)\right\} \frac{\sin \theta}{2 c(c-w \cos \theta)^{2}} \frac{\widetilde{P}}{\rho c} \cdot(39
\end{aligned}
$$

In order to get the relations for the mode $u=-c$, one needs to substitute $\pi-\theta$ for $\theta$, and multiply the right hand side of Eq. (39) by $(-1)$.

Consider the limiting case $\theta=0$, when the wave vector $\mathbf{k}$ is directed along the vector of the relative velocity $\mathbf{w}$. From Eqs. (37)-(39) and Eq. (26) for the first sound mode $u=+c$ we obtain:

$\widetilde{v}_{n x}=\left[1+\frac{1}{2}\left(3 u_{G}+1\right)+\left(3 u_{G}+2\right) \frac{w}{c}+\frac{1}{2}\left(u_{G}+3\right) \frac{w^{2}}{c^{2}}\right] \widetilde{v}_{s}$,

$$
\widetilde{P}=\rho c \widetilde{v}_{s}
$$

$$
\frac{\widetilde{T}}{T}=\left[\left(3 u_{G}+1\right)-4 u_{G} \frac{w}{c}-\left(u_{G}+3\right) \frac{w^{2}}{c^{2}}\right] \frac{1}{2(c-w)} \widetilde{v}_{s} .
$$

For the mode $u=-c$ we get

$$
\tilde{v}_{n x}=\left[1+\frac{1}{2}\left(3 u_{G}+1\right)-\left(3 u_{G}+2\right) \frac{w}{c}+\frac{1}{2}\left(u_{G}+3\right) \frac{w^{2}}{c^{2}}\right] \widetilde{v}_{s},
$$

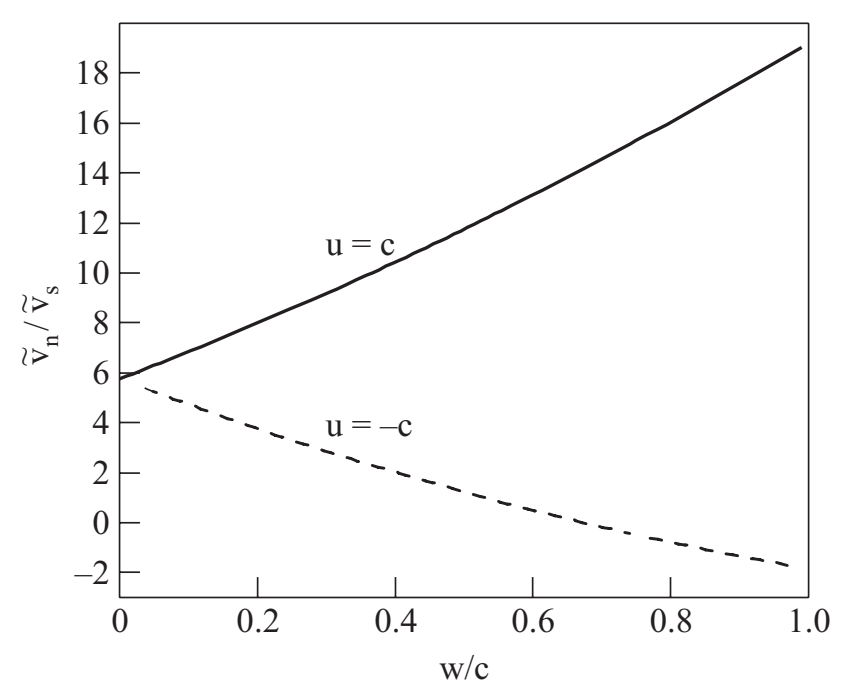

Fig. 2. The ratio of the amplitudes $\widetilde{v}_{n} / \widetilde{v}_{S}$ for the first sound modes $u=+c$ and $u=-c$, as a function of the relative velocity $w$, for phonon system at $\theta=0$, calculated from Eqs. (40) and (43).

$$
\begin{gathered}
\widetilde{P}=-\rho c \tilde{v}_{s} \\
\frac{\widetilde{T}}{T}=-\left[\left(3 u_{G}+1\right)+4 u_{G} \frac{w}{c}-\left(u_{G}+3\right) \frac{w^{2}}{c^{2}}\right] \frac{1}{2(c+w)} \widetilde{v}_{s} .
\end{gathered}
$$

In Fig. 2 we show the ratio of the amplitudes $\tilde{v}_{n} / \widetilde{v}_{s}$ for the first sound modes $u=+c$ and $u=-c$, as a function of the relative velocity $w$, for a phonon system at $\theta=0$, calculated from Eqs. (40) and (43). We see that, in the mode $u=-c$, when $w / c=0.67$, the normal component does not oscillate, and when $w / c>0.67$ the normal and superfluid components oscillate in antiphase. In Fig. 3 we show the ratio of the relative amplitudes $\widetilde{T}_{c} /\left(T \widetilde{v}_{S}\right)$ for the first sound modes $u=+c$ and $u=-c$ as a function of the relative velocity $w$, for a phonon system at $\theta=0$, calculated from Eqs. (42) and (45). We see that in the mode $u=+c$, when $w / c=0.63$ the temperature does not oscillate, and for $w / c>0.63$ the temperature and $\widetilde{\mathbf{v}}_{s}$ oscillate in antiphase.

We note, that for a phonon system in superfluid helium, the velocity of the normal fluid, in first sound, is many times that of the superfluid. For the case $w=0$ in Eq. (40)

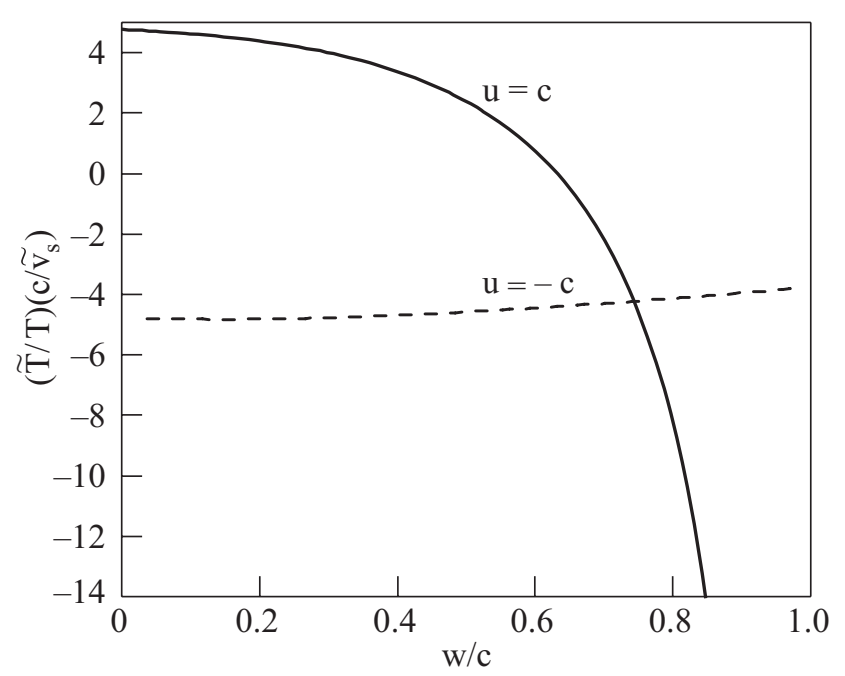

Fig. 3. The ratio of the relative amplitudes $\widetilde{T}_{c} /\left(\widetilde{v}_{S}\right)$ for the first sound modes $u=+c$ and $u=-c$ as a function of the relative velocity $w$, for phonon system at $\theta=0$, calculated from Eqs. (42) and (45). 


$$
\widetilde{v}_{n x}=\left[1+\frac{1}{2}\left(3 u_{G}+1\right)\right] \widetilde{v}_{s} \approx 6 \widetilde{v}_{s}
$$

So, in first sound $\widetilde{v}_{n} \neq \widetilde{v}_{s}$. The same result can be obtained directly from the relations in Ref. 13 .

It is interesting to note that in spite of the small value of the coefficient of thermal expansion, the relations (24), (25) involve the ratio between the small values of the thermal expansion coefficient $\alpha$ and the entropy of phonon gas $S$. This ratio is temperature independent in the phonon region. In detail:

$$
\alpha=-\frac{1}{\rho}\left(\frac{\partial \rho}{\partial T}\right)_{P, w=0}=\rho\left(\frac{\partial}{\partial T} \frac{1}{\rho}\right)_{P, w=0}=-\rho\left(\frac{\partial}{\partial P} \frac{S}{\rho}\right)_{T, w=0} .
$$

From this general equation for the phonon system we get

$$
\alpha_{\mathrm{ph}}=\frac{2 \pi^{2}}{45} \frac{3 u_{G}+1}{\hbar^{3} c^{5} \rho} T^{3} .
$$

It follows from Eq. (42) (or (45)) that the relative oscillations of temperature are not small compared to the relative oscillation of $\widetilde{v}_{s} / c$, even at $w=0$.

There are two reasons why, in the first sound mode $u=+c$ at $w \leq c$, the relative values of the temperature oscillation can reach large values in comparison with relative oscillations $\tilde{v}_{s} / c$ in spite of small value of thermal expansion coefficient. Firstly, the thermal expansion coefficient and entropy occur in the ratio $\alpha / S$, and, secondly, in the mode $u=+c$, when the relative velocity becomes $w \approx c$, the temperature oscillation becomes very large. The relation between the amplitudes in first sound in phonon systems, when $w$ is not very small, strongly depends on the angle $\theta$ between the wave vector $\mathbf{k}$ and the relative velocity vector $\mathbf{w}$. This follows from Eqs. (37)-(39). In the case $\theta=\pi / 2$ we get for both modes $u= \pm c$ :

$$
\begin{gathered}
\widetilde{v}_{n y}=\left[1+\frac{1}{2}\left(3 u_{G}+1\right)-\left(u_{G}+1\right) \frac{w^{2}}{2 c^{2}}\right] \widetilde{v}_{s}, \\
\widetilde{v}_{n x}=\widetilde{w}_{x}=\left[\left(u_{G}-1\right)+\left(u_{G}+1\right) \frac{w^{2}}{c^{2}}\right] \frac{w}{2 c} \widetilde{v}_{s}, \\
\widetilde{P}=\rho c \widetilde{v}_{s}, \\
\frac{\widetilde{T}}{T}=\left[\left(3 u_{G}+1\right)+\left(u_{G}+1\right) \frac{w^{2}}{c^{2}}\right] \frac{1}{2 c} \widetilde{v}_{s} .
\end{gathered}
$$

In Fig. 4 we show the ratio of the amplitudes, $\widetilde{v}_{n y} / \widetilde{v}_{s}$ and $\widetilde{v}_{n x} / \widetilde{v}_{s}$, for the first sound mode $u=+c$, as a function of the relative velocity $w$, for a phonon system at $\theta=\pi / 2$, calculated from Eqs. (49) and (50). We see that the normal fluid does not only oscillate in the longitudinal direction, but that there are also transverse oscillations. In Fig. 5 we

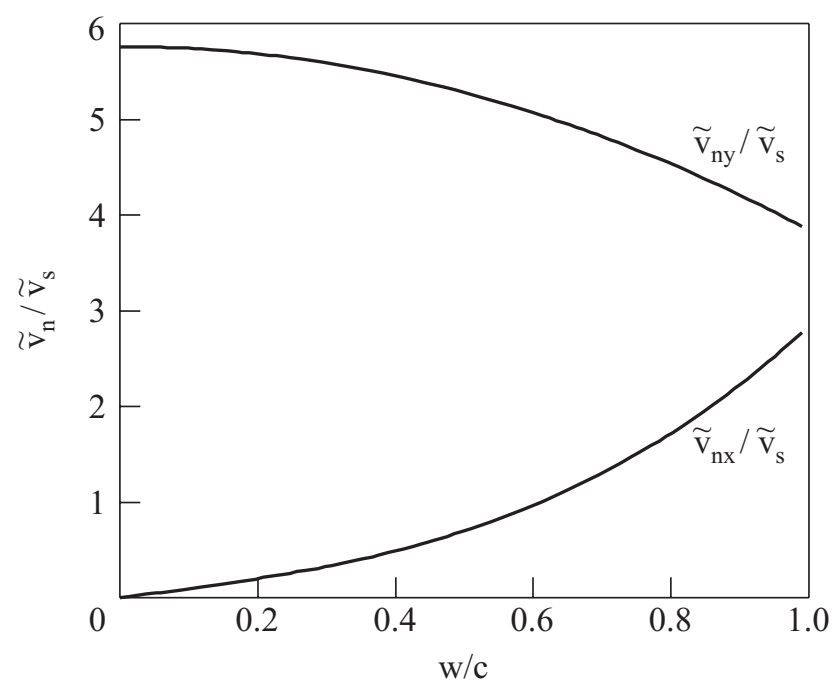

Fig. 4. The ratio of the amplitudes, $\widetilde{v}_{n y} / \widetilde{v}_{S}$ and $\widetilde{v}_{n x} / \widetilde{v}_{s}$, for the first sound mode $u=+c$, as a function of the relative velocity $w$, for phonon system at $\theta=\pi / 2$, calculated from Eqs. (49) and (50).

show the ratio of the relative amplitudes $\widetilde{T}_{c} /\left(T \widetilde{v}_{S}\right)$ for the first sound modes $u= \pm c$ as a function of the relative velocity $w$, for a phonon system at $\theta=\pi / 2$, calculated from Eq. (52). We see that as $w / c$ increases, the amplitude of the temperature oscillations increases.

At $w=0$, when the phonon system has no definite direction, we find, as expected, that the oscillations of the vector variables are longitudinal, $\widetilde{v}_{n x}=\widetilde{w}_{x}=0$, and Eqs. (49), (51), and (52) coincide with Eqs. (40), (41), and (42), respectively, at $w=0$.

In this paper, we have found the relationships between the amplitudes of the oscillating variables of first sound.

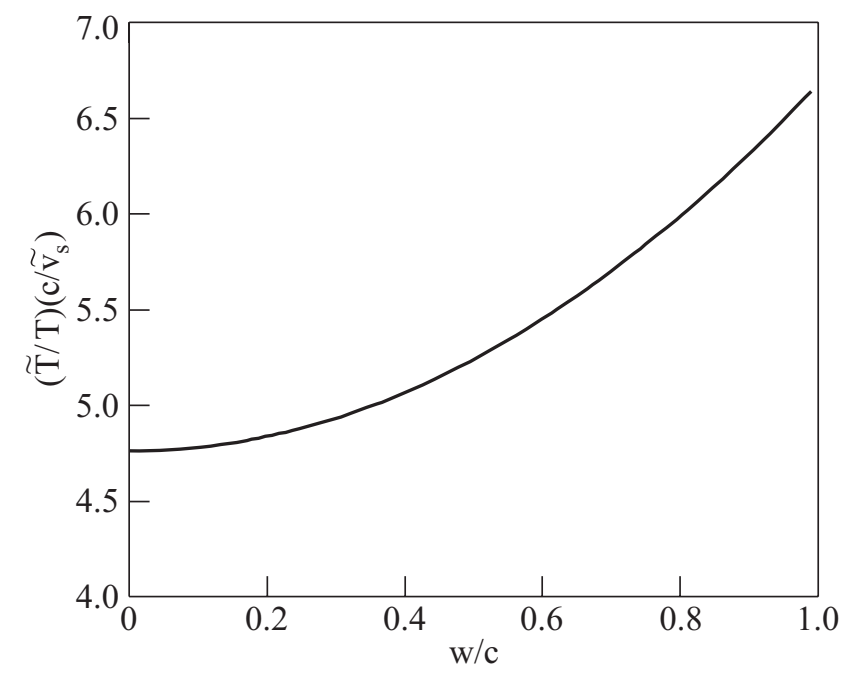

Fig. 5. The ratio of the relative amplitudes $\widetilde{T}_{c} /\left(T \widetilde{v}_{S}\right)$ for the first sound modes $u= \pm c$ as a function of the relative velocity $w$, for phonon system at $\theta=\pi / 2$, calculated from Eq. (52). 
Preliminary results for second sound were presented at QFS conference [16] and has been published in Ref. 17. A detailed study of second sound will be presented in our next paper.

We compare the results obtained in this paper to those for the isotropic case. In the case of second sound in the isotropic phonon system (i.e., $w=0$ ) (see Ref. 13) the relation between the hydrodynamic parameters is

$$
\begin{gathered}
\tilde{v}_{s}=\frac{2}{3 u_{G}-1} \frac{\rho_{n}}{\rho} \widetilde{v}_{n}, \\
\frac{\widetilde{P}}{\rho c}=\frac{3 u_{G}+1}{\sqrt{3}\left(3 u_{G}-1\right)} \frac{\rho_{n}}{\rho} \widetilde{v}_{n}, \\
\frac{\widetilde{T}}{T}=-\frac{2}{\sqrt{3}\left(3 u_{G}-1\right)} \frac{\widetilde{v}_{n}}{c} .
\end{gathered}
$$

In the relations (53)-(55) and below, we choose the independent variable $\widetilde{v}_{n}$ because the superfluid velocity $\widetilde{v}_{s}$ practically does not oscillate, see Eq. (53). The relations (53) and (55) should be compared with the respective relations for first sound in an anisotropic phonon system. For typical experimental values from Refs. 1, 5, 8, 9, for strongly anisotropic phonon systems $w / c=0.97$ and $T=0.05 \mathrm{~K}$.

For second sound in the isotropic phonon system, for the same density of normal component as in anisotropic case (i.e., at $T=0.42 \mathrm{~K}$ ), it follows from Eqs. (53)-(55) that:

$$
\frac{\widetilde{v}_{s}}{\widetilde{v}_{n}}=1.0 \cdot 10^{-6}, \quad \frac{\widetilde{P}}{\rho c \widetilde{v}_{n}}=2.9 \cdot 10^{-6}, \quad \frac{\widetilde{T} / T}{\widetilde{v}_{n} / c}=-0.15
$$

For the mode $u=+c$ of first sound in a phonon system with $w / c=0.97$, it follows from Eqs. (40)-(42) at $\theta=0$ that:

$$
\frac{\widetilde{v}_{s}}{\widetilde{v}_{n}}=\frac{\widetilde{P}}{\rho c \widetilde{v}_{n}}=0.053, \quad \frac{\widetilde{T} / T}{\widetilde{v}_{n} / c}=-6.2,
$$

and for the first sound mode $u=-c$, in an anisotropic system with $w / c=0.97$ at $\theta=0$, it follows from Eqs. (43)-(45) that:

$$
\frac{\widetilde{v}_{s}}{\widetilde{v}_{n}}=-\frac{\widetilde{P}}{\rho c \widetilde{v}_{n}}=-0.59, \quad \frac{\widetilde{T} / T}{\widetilde{v}_{n} / c}=2.25
$$

For the first sound mode in the isotropic phonon system it follows from Eqs. (43)-(45) that:

$$
\frac{\widetilde{v}_{s}}{\widetilde{v}_{n}}=\frac{\widetilde{P}}{\rho c \widetilde{v}_{n}}=0.17, \quad \frac{\widetilde{T} / T}{\widetilde{v}_{n} / c}=0.83 .
$$

We see, second sound, in the isotropic phonon system, in accordance with (56) is mainly a temperature wave and a wave of $\widetilde{v}_{n}$, and pressure and the superfluid component practically do not oscillate. The normal and superfluid currents are not equal in a phonon system, and can have the same direction. For first sound in the isotropic case, it follows from (59) that the superfluid and normal velocities, pressure and temperature all oscillate, and the temperature oscillation is not small. As for the mode of first sound, in the anisotropic case, for the mode $u=+c$, mainly the normal velocity and temperature oscillate, and pressure and superfluid velocity practically do not oscillate (see (57)). At the same time, in the first sound mode $u=-c$, in a phonon system with $w / c=0.97$, in accordance with (58), all the variables oscillate with similar values.

\section{Conclusion}

A notable feature of a strongly anisotropic phonon system in superfluid helium, created in experiments $[1,5,8,9]$, is the large value of the relative velocity $\mathbf{w}$, of the superfluid and normal components. The sound modes in stationary $(w=0)$ helium and for the case of small $w$, have been studied for many years, but the analysis of sound propagation at arbitrary $w$ has not been done until now.

In this paper the general dispersion equation for first and second sounds in superfluid helium is found, at an arbitrary thermodynamically stable value of $w$. It was shown, that in the limit of small contribution of thermal excitations, $\rho_{n} / \rho<<1$, at arbitrary value of $w$ in superfluid helium, the first sound modes can be excited with the dispersion law $\omega= \pm c k$. The general relation between the amplitudes of the oscillating variables in the first sound mode, is derived. In spite of «isotropic type» of dispersion law for the first sound modes, the relations between the amplitudes of the oscillating variables, strongly depend on the kind of mode (either $\omega=+c k$ or $\omega=-c k$ ) and on the angle between the velocity $\mathbf{w}$ and the wave vector $\mathbf{k}$. In the limiting case $w=0$, the general relations for the amplitudes of the oscillating variables is presented in Ref. 13.

The relation between the amplitudes of oscillating variables in first sound are studied in detail for the case of an anisotropic phonon system with arbitrary $\mathbf{w}$. This condition is very important in practice because high values of $\mathbf{w}$ are realized in phonon pulses propagating in superfluid helium $[1,5,8,9]$.

It is shown, that for strongly anisotropic phonon systems, as created in experiments, the amplitude of superfluid velocity, pressure, and temperature at a given oscillation amplitude of the normal velocity can be the same order of magnitude as the corresponding relations in a wave of second sound in the isotropic phonon system, and even exceed them. It should be noted, that even in the isotropic $(w=0)$ phonon system, the oscillation of velocities 
of superfluid $\widetilde{v}_{S}$ and normal $\widetilde{v}_{n}$ components do not equal one to another: $\widetilde{v}_{s} \neq \widetilde{v}_{n}$, and

$$
\widetilde{v}_{n}=\left[1+\left(3 u_{G}+1\right) / 2\right] \widetilde{v}_{s} \approx 6 \widetilde{v}_{s} .
$$

At $w \neq 0$ the inequality between $\widetilde{v}_{s}$ and $\widetilde{v}_{n}$ become stronger, see Fig. 2.

We should note here, that in the phonon region, the amplitude of the relative temperature (38) in first sound modes turns out not to be small in comparison with the relative oscillation amplitude of the normal (or super- fluid) component and strongly grows with $w$ when $w$ is close to $c$ for the mode $\omega=+c k$, see Fig. 3 .

So, the sound modes in superfluid helium, at nonzero values of the relative motion $w$, possess unusual properties, which are most apparent at large values of $w$. The authors hope that the relations between the amplitudes of the oscillating variables in the sound modes of superfluid helium when there is relative motion of normal fluid and superfluid components, obtained in this paper, will stimulate new experiments to study sound modes in anisotropic quasiparticle systems of superfluid helium.

\section{Appendix A}

The general dispersion equation for first and second sounds in superfluid helium at arbitrary value $w$ can be presented as follows

$$
a_{4} u^{4}+a_{3} u^{3}+a_{2} u^{2}+a_{1} u+a_{0}=0
$$

Here we introduce notations

$$
a_{4}=\rho_{n} \rho^{4}\left[\Gamma w^{2}+\rho_{n} \rho\left(\alpha^{2} c^{2}-\frac{\partial \sigma}{\partial T}\right)\right]
$$

where

$\Gamma=\rho_{n} \rho c^{2} \frac{\partial \rho_{n}}{\partial P} \alpha^{2}-\rho_{n} \rho \frac{\partial \rho_{n}}{\partial P} \frac{\partial \sigma}{\partial T}+\rho c^{2} \alpha^{2} \frac{\partial \rho_{n}}{\partial\left(w^{2} / 2\right)}+\left(\frac{\partial \rho_{n}}{\partial T}\right)^{2}-\rho \frac{\partial \sigma}{\partial T} \frac{\partial \rho_{n}}{\partial\left(w^{2} / 2\right)}+2 \rho c^{2} \alpha \frac{\partial \rho_{n}}{\partial T} \frac{\partial \rho_{n}}{\partial P}+\rho^{2} c^{2}\left(\frac{\partial \rho_{n}}{\partial P}\right)^{2} \frac{\partial \sigma}{\partial T}$,

and

$$
\sigma=S / \rho
$$

$a_{3}=2 \rho_{n} \rho^{3} w \cos (\theta)\left[\left(-\rho+2 \rho_{n}\right) \Gamma w^{2}-\rho\left(-2 \rho_{n}{ }^{2} c^{2} \alpha^{2}+2 \rho_{n}{ }^{2} \frac{\partial \sigma}{\partial T}+\rho \sigma \frac{\partial \rho_{n}}{\partial T}+\rho^{2} c^{2} \sigma \frac{\partial \rho_{n}}{\partial P} \alpha+2 \rho_{n} \rho c^{2} \alpha^{2}-2 \rho_{n} \rho \frac{\partial \sigma}{\partial T}\right)\right]$

$$
a_{2}=\rho^{2} w^{2} \cos ^{2}(\theta) a_{22}+\rho^{4} a_{20},
$$

where

$$
\begin{aligned}
& a_{22}=\rho_{n}\left(\rho^{2}+6 \rho_{n}^{2}-6 \rho_{n} \rho\right) \Gamma w^{2}-\rho\left[-c^{2} \sigma^{2}\left(\frac{\partial \rho_{n}}{\partial P}\right)^{2} \rho^{4}-4 \rho^{3} \rho_{n} c^{2} \sigma \frac{\partial \rho_{n}}{\partial P} \alpha+\rho^{3} \frac{\partial \rho_{n}}{\partial\left(w^{2} / 2\right)} \sigma^{2}+\right. \\
& +\rho^{3} \rho_{n} \sigma^{2} \frac{\partial \rho_{n}}{\partial P}+2 \rho^{2} \rho_{n}^{2} c^{2} \frac{\partial \rho_{n}}{\partial P} \frac{\partial \sigma}{\partial T}+4 \rho^{2} \rho_{n}^{2} \frac{\partial \sigma}{\partial T}-4 \rho^{2} \rho_{n} \sigma \frac{\partial \rho_{n}}{\partial T}+4 \rho^{2} \rho_{n}^{2} c^{2} \sigma \frac{\partial \rho_{n}}{\partial P} \alpha- \\
& \left.-4 \rho^{2} \rho_{n}^{2} c^{2} \alpha^{2}+4 \rho \rho_{n}^{2} \sigma \frac{\partial \rho_{n}}{\partial T}-12 \rho \rho_{n}^{3} \frac{\partial \sigma}{\partial T}+2 \rho \rho_{n}^{2} c^{2} \alpha \frac{\partial \rho_{n}}{\partial T}+12 \rho \rho_{n}^{3} c^{2} \alpha^{2}+6 \rho_{n}^{4} \frac{\partial \sigma}{\partial T}-6 \rho_{n}^{4} c^{2} \alpha^{2}\right],
\end{aligned}
$$

and

$$
\begin{aligned}
a_{20}= & {\left[-c^{2} \sigma^{2}\left(\frac{\partial \rho_{n}}{\partial P}\right)^{2} \rho^{3}+\rho^{2} \rho_{n} \sigma^{2} \frac{\partial \rho_{n}}{\partial P}+\rho^{2} \frac{\partial \rho_{n}}{\partial\left(w^{2} / 2\right)} \sigma^{2}+\rho^{2} c^{2} \rho_{n}\left(\frac{\partial \rho_{n}}{\partial P}\right)^{2} \sigma^{2}-2 \rho^{2} \rho_{n} c^{2} \sigma \frac{\partial \rho_{n}}{\partial P} \alpha-\right.} \\
& -2 \rho \rho_{n} \sigma \frac{\partial \rho_{n}}{\partial T}-\rho \rho_{n}^{2} \sigma^{2} \frac{\partial \rho_{n}}{\partial P}+\rho \rho_{n}^{2} \frac{\partial \sigma}{\partial T}-\rho \rho_{n}^{2} c^{2} \frac{\partial \rho_{n}}{\partial P} \frac{\partial \sigma}{\partial T}+\rho c^{2} \rho_{n} \frac{\partial \sigma}{\partial T} \frac{\partial \rho_{n}}{\partial\left(w^{2} / 2\right)}-
\end{aligned}
$$




$$
\begin{aligned}
-\rho \rho_{n} \sigma^{2} \frac{\partial \rho_{n}}{\partial\left(w^{2} / 2\right)}+2 \rho \rho_{n}^{2} c^{2} \sigma & \left.\frac{\partial \rho_{n}}{\partial P} \alpha-\rho \rho_{n}^{2} c^{2} \alpha^{2}-c^{2} \rho_{n}\left(\frac{\partial \rho_{n}}{\partial T}\right)^{2}+2 \rho_{n}^{2} \sigma \frac{\partial \rho_{n}}{\partial T}-2 \rho_{n}^{2} c^{2} \alpha \frac{\partial \rho_{n}}{\partial T}\right] w^{2}+ \\
& +\rho_{n} \rho\left(\rho_{n} c^{2} \frac{\partial \sigma}{\partial T}-\rho_{n} \sigma^{2}+\sigma^{2} \rho\right) ; \\
a_{1} & =2 \rho_{n} \rho w^{3} \cos ^{3}(\theta) a_{13}+2 \rho_{n} \rho^{3} w \cos (\theta) a_{11},
\end{aligned}
$$

where

$$
\begin{gathered}
a_{13}=\rho_{n}\left(\rho-2 \rho_{n}\right)\left(\rho-\rho_{n}\right) \Gamma w^{2}-\rho\left(\rho^{3} c^{2} \sigma \frac{\partial \rho_{n}}{\partial P} \frac{\partial \rho_{n}}{\partial T}-\rho^{3} \rho_{n} c^{2} \sigma \frac{\partial \rho_{n}}{\partial P} \alpha+\rho^{3} c^{2} \alpha \sigma \frac{\partial \rho_{n}}{\partial\left(w^{2} / 2\right)}-2 \rho^{3} \rho_{n} c^{2} \frac{\partial \rho_{n}}{\partial P} \frac{\partial \sigma}{\partial T}-\right. \\
-4 \rho^{2} \rho_{n}^{2} c^{2} \alpha^{2}-2 \rho^{2} \rho_{n} c^{2} \alpha \frac{\partial \rho_{n}}{\partial T}+4 \rho^{2} \rho_{n}^{2} \frac{\partial \sigma}{\partial T}+2 \rho^{2} \rho_{n}^{2} c^{2} \frac{\partial \rho_{n}}{\partial P} \frac{\partial \sigma}{\partial T}-2 \rho^{2} \rho_{n} \sigma \frac{\partial \rho_{n}}{\partial T}+\rho^{2} \rho_{n}^{2} c^{2} \sigma \frac{\partial \rho_{n}}{\partial P} \alpha+ \\
\left.+2 \rho \rho_{n}^{2} c^{2} \alpha \frac{\partial \rho_{n}}{\partial T}+6 \rho \rho_{n}^{3} c^{2} \alpha^{2}-6 \rho \rho_{n}^{3} \frac{\partial \sigma}{\partial T}+\rho \rho_{n}^{2} \sigma \frac{\partial \rho_{n}}{\partial T}+2 \rho_{n}^{4} \frac{\partial \sigma}{\partial T}-2 \rho_{n}^{4} c^{2} \alpha^{2}\right),
\end{gathered}
$$

and

$$
\begin{gathered}
a_{11}=\left[2\left(\rho-\rho_{n}\right)\left(-\frac{\partial \rho_{n}}{\partial T} \rho_{n}+\rho c^{2} \alpha \frac{\partial \rho_{n}}{\partial\left(w^{2} / 2\right)}+\rho c^{2} \frac{\partial \rho_{n}}{\partial P} \frac{\partial \rho_{n}}{\partial T}\right) \sigma-\rho^{2} \rho_{n} c^{2} \frac{\partial \rho_{n}}{\partial P} \frac{\partial \sigma}{\partial T}-\rho^{2} c^{2} \frac{\partial \sigma}{\partial T} \frac{\partial \rho_{n}}{\partial\left(w^{2} / 2\right)}-\rho \rho_{n}^{2} c^{2} \alpha^{2}+\right. \\
\left.+\rho \rho_{n}^{2} \frac{\partial \sigma}{\partial T}+2 \rho c^{2} \rho_{n} \frac{\partial \sigma}{\partial T} \frac{\partial \rho_{n}}{\partial\left(w^{2} / 2\right)}-2 c^{2} \rho_{n}\left(\frac{\partial \rho_{n}}{\partial T}\right)^{2}-2 \rho_{n}^{2} c^{2} \alpha \frac{\partial \rho_{n}}{\partial T}+\rho c^{2}\left(\frac{\partial \rho_{n}}{\partial T}\right)^{2}\right] w^{2}+ \\
+c^{2} \rho\left(3 \rho_{n} \rho \alpha+\rho \frac{\partial \rho_{n}}{\partial T}-2 \alpha \rho_{n}^{2}\right) \sigma+2 \rho_{n} c^{2} \frac{\partial \sigma}{\partial T} \rho\left(-\rho+\rho_{n}\right) \\
a_{0}=\rho_{n}^{2} w^{4} \cos ^{4}(\theta) a_{04}+\rho^{2} w^{2} \cos ^{2}(\theta) a_{02}+\left(\rho-\rho_{n}\right) \rho^{4} a_{00}
\end{gathered}
$$

where

$$
\begin{aligned}
& a_{04}=\rho_{n}\left(\rho-\rho_{n}\right)^{2} \Gamma w^{2}-\rho\left[\rho^{3} c^{2} \frac{\partial \sigma}{\partial T} \frac{\partial \rho_{n}}{\partial\left(w^{2} / 2\right)}-3 \rho^{3} \rho_{n} c^{2} \frac{\partial \rho_{n}}{\partial P} \frac{\partial \sigma}{\partial T}+4 \rho^{2} \rho_{n}^{2} \frac{\partial \sigma}{\partial T}-\rho^{2} c^{2}\left(\frac{\partial \rho_{n}}{\partial T}\right)^{2}-4 \rho^{2} \rho_{n}^{2} c^{2} \alpha^{2}-\right. \\
& \left.-4 \rho^{2} \rho_{n} c^{2} \alpha \frac{\partial \rho_{n}}{\partial T}+2 \rho^{2} \rho_{n}^{2} c^{2} \frac{\partial \rho_{n}}{\partial P} \frac{\partial \sigma}{\partial T}+4 \rho \rho_{n}^{3} c^{2} \alpha^{2}-4 \rho \rho_{n}^{3} \frac{\partial \sigma}{\partial T}+2 \rho \rho_{n}^{2} c^{2} \alpha \frac{\partial \rho_{n}}{\partial T}-\rho_{n}^{4} c^{2} \alpha^{2}+\rho_{n}^{4} \frac{\partial \sigma}{\partial T}\right] \text {, } \\
& a_{02}=\left\{-\rho_{n} \rho\left(\rho-\rho_{n}\right)^{2}\left[-\frac{\partial \rho_{n}}{\partial P} \rho_{n}+\rho c^{2}\left(\frac{\partial \rho_{n}}{\partial P}\right)^{2}-\frac{\partial \rho_{n}}{\partial\left(w^{2} / 2\right)}\right] \sigma^{2}-2\left(-\rho+\rho_{n}\right) \rho_{n}\left(-\rho_{n}^{2} \frac{\partial \rho_{n}}{\partial T}+\rho_{n}^{2} \rho c^{2} \frac{\partial \rho_{n}}{\partial P} \alpha+\right.\right. \\
& \left.+2 \rho_{n} \rho c^{2} \alpha \frac{\partial \rho_{n}}{\partial\left(w^{2} / 2\right)}+2 \rho_{n} \rho c^{2} \frac{\partial \rho_{n}}{\partial P} \frac{\partial \rho_{n}}{\partial T}-\rho_{n} \rho^{2} c^{2} \frac{\partial \rho_{n}}{\partial P} \alpha-\rho^{2} c^{2} \alpha \frac{\partial \rho_{n}}{\partial\left(w^{2} / 2\right)}-\rho^{2} c^{2} \frac{\partial \rho_{n}}{\partial P} \frac{\partial \rho_{n}}{\partial T}\right) \sigma+ \\
& +\rho_{n}\left[\rho_{n}^{3} \rho c^{2} \frac{\partial \rho_{n}}{\partial P} \frac{\partial \sigma}{\partial T}-2 \rho_{n}^{3} c^{2} \alpha \frac{\partial \rho_{n}}{\partial T}+\rho_{n}^{3} \rho \frac{\partial \sigma}{\partial T}-\rho_{n}^{3} \rho c^{2} \alpha^{2}-3 \rho^{2} \rho_{n}^{2} c^{2} \frac{\partial \rho_{n}}{\partial P} \frac{\partial \sigma}{\partial T}+3 \rho_{n}^{2} \rho c^{2} \frac{\partial \sigma}{\partial T} \frac{\partial \rho_{n}}{\partial\left(w^{2} / 2\right)}-3 \rho_{n}^{2} c^{2}\left(\frac{\partial \rho_{n}}{\partial T}\right)^{2}+\right. \\
& \left.\left.+3 \rho_{n} \rho c^{2}\left(\frac{\partial \rho_{n}}{\partial T}\right)^{2}-3 \rho_{n} \rho^{2} c^{2} \frac{\partial \sigma}{\partial T} \frac{\partial \rho_{n}}{\partial\left(w^{2} / 2\right)}+\rho^{3} \rho_{n} c^{2} \frac{\partial \rho_{n}}{\partial P} \frac{\partial \sigma}{\partial T}-\rho^{2} c^{2}\left(\frac{\partial \rho_{n}}{\partial T}\right)^{2}+\rho^{3} c^{2} \frac{\partial \sigma}{\partial T} \frac{\partial \rho_{n}}{\partial\left(w^{2} / 2\right)}\right]\right\} w^{2}+ \\
& +\rho\left(-4 \rho_{n}^{3} \rho+\rho_{n}^{4}+\rho^{3} c^{2} \frac{\partial \rho_{n}}{\partial\left(w^{2} / 2\right)}+4 \rho^{2} \rho_{n}^{2}-3 \rho^{3} \rho_{n} c^{2} \frac{\partial \rho_{n}}{\partial P}+2 \rho^{2} \rho_{n}^{2} c^{2} \frac{\partial \rho_{n}}{\partial P}\right) \sigma^{2}+ \\
& +4 c^{2} \rho_{n} \rho\left(-\rho+\rho_{n}\right)\left(-\alpha \rho_{n}^{2}+2 \rho_{n} \rho \alpha+\rho \frac{\partial \rho_{n}}{\partial T}\right) \sigma+\rho \rho_{n}^{2} c^{2} \frac{\partial \sigma}{\partial T}\left(-\rho+\rho_{n}\right)\left(3 \rho_{n}-4 \rho\right), \\
& a_{00}=\left[\left(-\rho_{n}^{2}+\rho_{n} \rho c^{2} \frac{\partial \rho_{n}}{\partial P}-c^{2} \frac{\partial \rho_{n}}{\partial\left(w^{2} / 2\right)} \rho\right) \sigma^{2}+2 c^{2} \rho_{n}\left(\frac{\partial \rho_{n}}{\partial T}+\rho_{n} \alpha\right) \sigma-\frac{\partial \sigma}{\partial T} c^{2} \rho_{n}^{2}\right] w^{2}-\rho_{n} \rho c^{2} \sigma^{2} \text {. }
\end{aligned}
$$




\section{Appendix B}

$$
\begin{gathered}
C=\left(2 \frac{\partial \ln S}{\partial T}-\frac{\partial \ln \rho_{n}}{\partial T}\right) w^{2} \cos ^{3} \theta+\left[\Delta_{1} \rho w^{2}-\frac{\partial \ln S}{\partial T}+\frac{\rho S}{\rho_{n}}\left(\frac{\partial \ln \rho_{n}}{\partial P}-2 \frac{\partial \ln S}{\partial P}\right)\right] c w \cos ^{2} \theta+ \\
+\left(-2 \Delta_{1} \rho c^{2} w^{2}+\Delta_{2}\right) \cos \theta+\left(\Delta_{1} \rho c^{2}+\frac{\rho S}{\rho_{n}} \frac{\partial \ln S / \rho_{n}}{\partial P}-\frac{\partial \ln S / \rho_{n}}{\partial T}\right) c w,
\end{gathered}
$$

where we denote

$$
\begin{gathered}
\Delta_{1}=\frac{\partial \ln \rho_{n}}{\partial P} \frac{\partial \ln S}{\partial T}-\frac{\partial \ln S}{\partial P} \frac{\partial \ln \rho_{n}}{\partial T}, \Delta_{2}=\frac{S}{\rho_{n}}\left(\frac{\partial \ln S}{\partial P} \rho c^{2}-1\right) \\
D=\left[\Delta_{3} w^{2}-4 \frac{S}{\rho_{n}} \frac{\partial \ln S / \rho_{n}}{\partial T}-\left(\frac{S}{\rho_{n}}\right)^{2} \frac{\partial \ln \rho_{n}}{\partial\left(w^{2} / 2\right)}\right] w^{2} \cos ^{2} \theta-\left[\Delta_{3} w^{2}-\frac{S}{\rho_{n}}\left(2 \frac{\partial \ln S}{\partial T}-\frac{\partial \ln \rho_{n}}{\partial T}\right)\right] 2 c w \cos \theta+ \\
+\left[\Delta_{3} c^{2}+\frac{S}{\rho_{n}}\left(\frac{\partial \ln S}{\partial T}-2 \frac{\partial \ln \rho_{n}}{\partial T}+\frac{\partial \ln \rho_{n}}{\partial\left(w^{2} / 2\right)} \frac{S}{\rho_{n}}\right)\right] w^{2}+\frac{S}{\rho_{n}}\left(\frac{S}{\rho_{n}}-\frac{\partial \ln S}{\partial T} c^{2}\right),
\end{gathered}
$$

where we use the following shortcut

$$
\begin{gathered}
\Delta_{3}=\left(\frac{\partial \ln \rho_{n}}{\partial T}\right)^{2}-\frac{S}{\rho_{n}} \frac{\partial \ln S}{\partial T} \frac{\partial \ln \rho_{n}}{\partial\left(w^{2} / 2\right)} ; \\
E=\left(\frac{S}{\rho_{n}} \frac{\partial \ln \rho_{n}}{\partial\left(w^{2} / 2\right)}-2 \frac{\partial \ln \rho_{n}}{\partial T}\right) w^{3} \cos ^{3} \theta+\left[\Delta_{4} \rho w^{2}+2 \frac{S \rho}{\rho_{n}}\left(2 \frac{\partial \ln S}{\partial P}-\frac{\partial \ln \rho_{n}}{\partial P}\right)+\frac{\partial \ln \rho_{n}}{\partial T}\right] c w^{2} \cos ^{2} \theta+ \\
+\left\{-2 \Delta_{4} \rho c^{2} w^{2}+\frac{S}{\rho_{n}}\left[1+\left(\frac{\partial \ln \rho_{n}}{\partial P}-4 \frac{\partial \ln S}{\partial P}\right) \rho c^{2}\right]\right\} w \cos (\theta)+ \\
+\left(\Delta_{4} \rho c^{2}-\frac{S \rho}{\rho_{n}} \frac{\partial \ln S / \rho_{n}}{\partial P}+\frac{\partial \ln \rho_{n}}{\partial T}-\frac{S}{\rho_{n}} \frac{\partial \ln \rho_{n}}{\partial\left(w^{2} / 2\right)}\right) c w^{2}+\Delta_{2} c,
\end{gathered}
$$

where

$$
\Delta_{4}=\frac{S}{\rho_{n}} \frac{\partial \ln S}{\partial P} \frac{\partial \ln \rho_{n}}{\partial\left(w^{2} / 2\right)}-\frac{\partial \ln \rho_{n}}{\partial P} \frac{\partial \ln \rho_{n}}{\partial T} .
$$

\section{Acknowledgements}

We express our gratitude to EPSRC of the UK (grant $\mathrm{EP} / \mathrm{F}$ 019157/1) for support of this work.

1. A.F.G. Wyatt, N.A. Lockerbie, and R.A. Sherlock, Phys. Rev. Lett. 33, 1425 (1974).

2. A.F. Andreev and L.A. Melnikovsky, JETP Lett. 78, 574 (2003) [Pis'ma Zh. Eksp. Teor. Fiz. 78, 1063 (2003)].

3. I.N. Adamenko, K.E. Nemchenko, V.A. Slipko, and A.F.G. Wyatt, Phys. Rev. Lett. 96, 065301 (2006).

4. I.N. Adamenko, K.E. Nemchenko, V.A. Slipko, and A.F.G. Wyatt, J. Phys.: Condens. Matter 18, 2805 (2006).

5. R. Vovk, C.D.H. Williams, and A.F.G. Wyatt, Phys. Rev. Lett. 91, 235302 (2003).

6. R.E. Grisenti and J.P. Toennies, Phys. Rev. Lett. 90, 234501 (2003).

7. J.A. Flaten, C.A. Lindesmith, and W. Zimmermann, J. Low Temp. Phys. 101, 743 (1995).
8. D.H.S. Smith, R.V. Vovk, C.D.H. Williams, and A.F.G. Wyatt, New J. Phys. 8, 128 (2006).

9. D.H.S. Smith and A.F.G. Wyatt, accepted for publication in Phys. Rev. B.

10. I.M. Khalatnikov, Pis'ma Zh. Eksp. Teor. Fiz. 30, 617 (1956).

11. R.G. Arkhipov, Z. Eksp. Teor. Phys. 59, 2055 (1970).

12. A.F. Andreev and L.A. Melnikovsky, J. Low Temp. Phys. 135, 411 (2004).

13. I.M. Khalatnikov, An Introduction to the Theory of Superfluidity, W.A. Benjamin (ed.), New York-Amserdam (1965).

14. S.J. Putterman, Superfluid Hydrodynamics, Horth Holland, Amsterdam (1974).

15. to be published.

16. International Symposium on Quantum Fluids and Solids QFS2006, Kyoto (2006).

17. I.N. Adamenko, K.E. Nemchenko, V.A. Slipko, and A.F.G. Wyatt, J. Low Temp. Phys. 148, 547 (2007). 